\title{
MIEDOS COMUNES EN NIÑOS Y ADOLESCENTES: RELACIÓN CON LA SENSIBILIDAD A LA ANSIEDAD, EL RASGO DE ANSIEDAD, LA AFECTIVIDAD NEGATIVA Y LA DEPRESIÓN
}

\author{
ROSA M. VALIENTE, BONIFACIO SANDÍN Y PALOMA CHOROT
}

Universidad Nacional de Educación a Distancia (UNED), Madrid

(Aceptado en enero de 2001)

\begin{abstract}
En el presente estudio hemos examinado las relaciones entre los miedos comunes y la sensibilidad a la ansiedad, el rasgo de ansiedad, la afectividad negativa y la depresión en una muestra no clínica de niños y adolescentes. Una amplia muestra $(N=1080)$ de niños y adolescentes completó las versiones españolas de los cuestionarios Childhood Anxiety Sensitivity Index (CASI; Silverman, Fleisig, Rabian \& Peterson, 1991), Fear Survey Schedule for Children-Revised (FSSC-R; Ollendick, 1983), y State-Trait Anxiety Inventory for Children (STAIC, Spielberger, 1970), así como también un cuestionario sobre depresión infantojuvenil (Sandín \& Valiente, 1996). En términos globales los resultados indicaron la existencia de: (1) correlaciones moderadas entre el miedo y las variables de sensibilidad a la ansiedad, rasgo de ansiedad y afectividad negativa; (2) correlaciones bajas entre el miedo y la depresión; y (3) superiores correlaciones (diferencias estadísticamente significativas) entre el miedo y la sensibilidad a la ansiedad que entre el miedo y las restantes variables. Estos datos apoyan la hipótesis de que la sensibilidad a la ansiedad podría ser considerada como una variable de vulnerabilidad hacia el miedo, más específica que el rasgo de ansiedad o la afectividad negativa. Los resultados son también discutidos en términos de la separación entre los constructos de miedo, sensibilidad a la ansiedad, ansiedad y depresión, así como también en relación con el más global constructo de afectividad negativa.
\end{abstract}

Palabras clave: Miedos en niños y adolescentes, sensibilidad a la ansiedad, rasgo de ansiedad, afectividad negativa, depresión.

\section{Common fears in children and adolescents: Their relationship to anxiety sensitivity, trait anxiety, negative affectivity, and depression}

In the present study, we examined the relations of common fears to anxiety sensitivity, trait anxiety, negative affectivity, and depression in a sample of nonclinical children and adolescents. A large sample of children and adolescents $(N=1080)$ completed the Spanish versions of the Childhood Anxiety Sensitivity Index (CASI; Silverman, Fleisig, Rabian \& Peterson, 1991), the Fear Survey Schedule for Children-Revised (FSSCR; Ollendick, 1983), the State-Trait Anxiety Inventory for Children (STAIC, Spielberger, 1970), and a measure of depression (Sandin \& Valiente, 1996). Overall, results showed: (1) moderate correlations between fear and anxiety sensitivity, trait anxiety, and negative affectivity; (2) modest correlations between fear and depression; and (3) fear correlated significantly higher with anxiety sensitivity than with the remaining measures. Findings support the hypothesis that anxiety sensitivity could be viewed as a variable of vulnerability to fear, more specific than trait anxiety or negative affectivity. Results were also discussed in terms of the uniqueness of the constructs of fear, anxiety sensitivity, anxiety and depression, as well as, in relation with the more global construct of negative affectivity.

Key words: Fears in children and adolescents, anxiety sensitivity, trait anxiety, negative affectivity, depression.

Correspondencia: Rosa M. Valiente, Facultad de Psicología, Universidad Nacional de Educación a Distancia, Ciudad Universitaria s/n, 28040 Madrid. Correo-e: rmvalien@psi.uned.es.
Agradecimientos: Deseamos agradecer a la psicóloga Aurora Tabar su ayuda en la aplicación de las pruebas en los diferentes centros educativos de Navarra. 


\section{INTRODUCCIÓN}

Si bien se asume la existencia de cierta predisposición filogenética para adquirir los miedos comunes, existen también diferencias individuales que parecen influir en la adquisición y mantenimiento de los mismos, pudiendo interaccionar con otras variables de tipo ambiental (p.ej., entorno familiar, pautas de crianza, sucesos vitales, etc.), y modular la evolución normal o patológica de los temores infantojuveniles. Aspectos constitucionales diversos como los determinados por la herencia, la transmisión de patrones familiares, las variables de personalidad (p.ej., el neuroticismo o el rasgo de ansiedad) la sensibilidad a la ansiedad, la reactividad psicofisiológica, la diferenciación sexual del cerebro, o los ritmos psicobiológicos, entre otros, podrían explicar en cierto modo por qué algunos niños son más miedosos que otros en la misma etapa evolutiva, o por qué en ellos no desaparece la respuesta normal de miedo ante un estímulo concreto cuando, por la edad, cabría esperar que se redujera.

Aunque suele aceptarse que los niños más ansiosos tienden también a presentar mayores niveles de miedo, son muy pocos los estudios que han analizado de forma específica esta cuestión en población infantojuvenil. Tal vez una de las causas obedece a que el miedo y la ansiedad con frecuencia se confunden en los estudios infantojuveniles. Sin embargo, la ausencia de diferenciación entre ambos constructos a veces ha sido criticada en el ámbito de la psicopatología del desarrollo (p.ej., Gullone, King y Ollendick, 2001). Como han señalado estos autores, el miedo y la ansiedad claramente se solapan en aspectos afectivos y fisiológicos (aprensión, temblor, sudoración, etc.); sin embargo, el miedo y la ansiedad son conceptualmente separables (véase Sandín, 1999a). La ansiedad también se ha diferenciado de la depresión en la población infantojuvenil (Boyd y Gullone, 1997), a pesar de que algunos autores hayan abogado, asumiendo una difícil diferenciación durante estas etapas del desarrollo, por un factor general de distress que engloba al miedo, la ansiedad y la depresión (véase King, Ollendick y Gullone, 1991). El modelo tripartito sobre la ansiedad y la depresión ha sido de gran ayuda para separar la ansiedad de la depresión; el modelo se basa en la existencia de un componente común a la ansiedad y la depresión (la afectividad negativa) y en componentes específicos para la ansiedad (hiperactivación) y la depresión (bajo afecto positivo) (véase Joiner, Catanzaro, Laurent, Sandín, y Blalock., 1996a). El modelo también ha sido probado en población de niños y adolescentes (p.ej., Joiner, Catanzaro y Laurent, 1996b; Chorpita, Albano y Barlow, 1998).

A pesar del interés que presenta el estudio de las relaciones entre el miedo, la ansiedad y la depresión en niños y adolescentes, así como de la diferenciación entre dichas emociones, son pocos los estudios que han abordado de forma directa esta cuestión. Los escasos trabajos que han estudiado este problema tienden a sugerir que el autoinforme de miedos correlaciona moderadamente con el rasgo de ansiedad y de forma más débil con la depresión (p.ej., Ollendick, Yule y Ollier, 1991; King, Gullone y Ollendick, 1992; Dong, Yang y Ollendick, 1994; Muris, Schmidt, Merckelbach y Schouten, 2001). Así por ejemplo, en el estudio de Ollendick et al. (1991) la correlación entre ansiedad y miedo $(r=0,64)$ fue superior a la correlación entre depresión y miedo $(r=0,31)$. De forma similar, Dong et al. (1994) informaron que el nivel de miedo estaba mínimamente relacionado con la depresión $(r=0,20)$ y moderadamente relacionado con la ansiedad $(r=0,38)$. Muris et al. (2001), por citar un estudio más reciente, encontra- 
ron, igualmente, una correlación moderada entre los niveles de miedo y ansiedad $(r=0,58)$ y una correlación más modesta entre el miedo y la depresión $(r=0,31)$.

Por otra parte, las correlaciones entre depresión y ansiedad suelen ser moderadas; en estos estudios las correlaciones entre ansiedad y depresión han sido similares o superiores a las correlaciones entre ansiedad y miedo (p.ej., $r=0,64$, en el estudio de Dong et al., 1994; $r=0,60$, en el estudio de Muris et al., 2001). Es decir, aunque tanto el miedo como la depresión se relacionan de modo importante con la ansiedad, la relación entre el miedo y la depresión parece más débil. Esta relación que se da entre las tres variables consideradas podría indicar que la ansiedad posee aspectos comunes al miedo y la depresión, características que, sin embargo, no son compartidas por estas dos últimas variables. Así, la ansiedad podría compartir con la depresión características de la afectividad negativa no compartidas con el miedo, mientras que, por otra parte, la ansiedad podría compartir con el miedo características de hiperactivación no compartidas con la depresión. Esta explicación, si bien es a grosso modo consistente con el modelo tripartito de la ansiedad y la depresión (Joiner et al., 1996a, 1996b; Barlow et al., 1998), supone una variación en el mismo, ya que implica una separación entre la ansiedad y el miedo. En este momento, que nosotros sepamos, dos trabajos han aportado datos a favor de esta caracterización tridimensional, i.e., una separación entre el miedo, la ansiedad y la depresión. El primero de ellos fue el publicado por Chorpita et al. (1998), basado una muestra de niños y adolescentes con trastornos de ansiedad y depresión. El segundo fue el estudio de Muris et al. (2001) con una muestra de niños y adolescentes no clínicos. En ambos trabajos, basados éstos en análisis factorial confirmatorio, se demostró que el modelo más adecuado (i.e., el que mejor se ajustaba a los datos) era el constituido por tres factores correlacionados (miedo, ansiedad y depresión saturarían en tres factores diferentes). Otras alternativas, como la consistente en uno (miedo, ansiedad y depresión saturando en un único factor) o dos factores (miedo $\mathrm{y}$ ansiedad saturando en un factor, depresión saturando en un segundo factor; o bien, ansiedad y depresión saturando en un factor, miedo saturando en un segundo factor), resultaron ser menos convincentes. Por tanto, parece que, aunque existe cierta relación entre el miedo, la ansiedad y la depresión, podríamos decir que los datos indican que, incluso para la población infantojuvenil, constituyen variantes emocionales diferenciables teórica y empíricamente.

Estos estudios sugieren que, al margen de las consideraciones diferenciales relativas a las tres variables indicadas, la ansiedad y la depresión podrían formar parte de un factor general de vulnerabilidad hacia los miedos, factor que se correspondería con el constructo de afectividad negativa (Valiente, 2001). Existen, sin embargo, otras posibles variables que podrían relacionarse con los miedos de forma más específica que la ansiedad, la depresión o la afectividad negativa. Una variable que ha sido designada como posible candidata en este sentido es la denominada sensibilidad a la ansiedad. Ésta ha sido definida como una variable de diferencias individuales consistente en una tendencia a experimentar miedo ante los síntomas de ansiedad (sobre todo, los síntomas fisiológicos), y constituye el rasgo, tendencia o predisposición a interpretar "catastróficamente» -es decir, como algo peligroso- esas sensaciones de ansiedad, especialmente las sensaciones de activación fisiológica (Taylor, 1995). La sensibilidad a la ansiedad es, así mismo, diferente del rasgo de 
ansiedad, ya que este último denota una tendencia a reaccionar con miedo ante cualquier estresor o estímulo ansiógeno (Sandín, Chorot y McNally, 2001). Aunque se ha propuesto como variable de vulnerabilidad hacia los trastornos de ansiedad en general, la sensibilidad a la ansiedad parece relacionarse mucho más específicamente con el miedo $\mathrm{y}$, especialmente, con el trastorno de pánico (McNally, 1994; Sandín, Chorot y McNally, 1996; Chorot, Sandín, Valiente, Santed y Romero, 1997), siendo hoy un concepto central para el diagnóstico de este trastorno.

A pesar de que algunos autores han cuestionado la relevancia de la sensibilidad a la ansiedad en la población infantojuvenil (Chorpita, Albano y Barlow, 1996), existe evidencia de que ésta posee una especial utilidad para pronosticar el riesgo de sufrir miedos, pánico y otros problemas de ansiedad (p.ej., Lau, Calamari y Waraczynski, 1996; Sandín, 1997; Chorpita y Daleiden, 2000; Silverman y Weems, 1999). En un estudio reciente con una muestra de niños de 9-11 años de edad, Sandín, Chorot, Valiente y Santed (en prensa) encontraron que la sensibilidad a la ansiedad se relacionaba con el nivel de miedos de forma más intensa que el rasgo de ansiedad, sugiriendo que la sensibilidad a la ansiedad en niños podía ser considerada como un constructo único de vulnerabilidad hacia el miedo. Los autores sugieren que, si bien el rasgo de ansiedad puede seguir siendo considerado como un factor de vulnerabilidad hacia los miedos, la sensibilidad a la ansiedad resulta ser una variable más estrechamente relacionada con el miedo $\mathrm{y}$, en consecuencia, podría constituir un mejor indicador de vulnerabilidad hacia el mismo.

Mediante el presente trabajo pretendemos, partiendo de una muestra amplia de participantes no clínicos, estudiar la relación entre los miedos y un conjunto rele- vante de factores de diferencias individuales potencialmente de predisposición a los mismos, esto es, la sensibilidad a la ansiedad, el rasgo de ansiedad, la afectividad negativa, y la depresión. Hipotetizamos que la sensibilidad a la ansiedad, el rasgo de ansiedad, el afecto negativo y la depresión deberían relacionarse de manera positiva con los niveles de miedo. Pronosticamos una relación más estrecha de los miedos con la sensibilidad a la ansiedad que con las restantes variables de diferencias individuales, y una relación más específica de los miedos con el rasgo de ansiedad y la afectividad negativa que con la depresión. Tales relaciones deberían mantenerse tanto para la intensidad como para la prevalencia de los miedos, y tanto para los niveles totales de miedo como para los niveles de las diferentes dimensiones (i.e., miedos a los animales, al fracaso y crítica, al peligro y muerte, a lo desconocido, y a las situaciones médicas).

\section{MÉTODO}

\section{Participantes}

La muestra fue de 1080 participantes normales (no clínicos ni con retraso mental), la cual estaba formada por 613 chicas y 467 chicos, con edades que oscilaban entre los 8 y los 18 años $(M=13,62, D T=$ 2,50 años). Los participantes poseían mayoritariamente niveles socioeconómicos medio o medio-bajo, y asistían regularmente a colegios públicos de enseñanza primaria o secundaria ubicados en la Comunidad de Navarra.

\section{Pruebas psicológicas y procedimiento}

Los participantes fueron evaluados en su propio centro docente, al cual asistían con regularidad durante el curso académico. 
De forma colectiva y supervisada por dos psicólogas se les aplicaron las siguientes pruebas psicométricas (junto a otras pruebas no incluidas en el presente estudio):

Cuestionario de Miedos para NiñosRevisado [Fear Survey Schedule for Children-Revised] (FSSC-R; Ollendick, 1983). Aplicamos la versión española de Chorot y Sandín (Sandín, 1997). La versión española, al igual que la versión en lengua inglesa, consta de 80 items de tres niveles de intensidad $(1,2$ y 3$)$, mediante los cuales puede establecerse una evaluación global de los miedos infantojuveniles. El nivel general de miedos se obtiene sumando los niveles de intensidad referidos para cada ítem. El nivel de prevalencia de los miedos se calcula sumando en número total de miedos que el sujeto estimó con la puntuación de máxima intensidad. El cuestionario incluye las 5 subescalas siguientes: (1) Miedos al fracaso y la crítica, (2) Miedos a pequeños animales y daños menores, (3) Miedos a los peligros físicos y muerte, (4) Miedos a lo desconocido, y (5) Miedos médicos. Estudios recientes con la versión española del FSSC-R han aportado datos psicométricos que garantizan una elevada consistencia interna y una estructura de cinco factores similar a la establecida para la versión inglesa del cuestionario (Sandín y Chorot, 1998; Sandín, Chorot, Valiente y Santed, 1998; Valiente, 2001).

Índice de Sensibilidad a la Ansiedad para Niños [Childhood Anxiety Sensitivity Index] (CASI; Silverman et al., 1991). Se aplicó la versión española de Sandín y Chorot (Sandín, 1997). Consta de 18 items que se puntúan según una escala Likert de 3 puntos: Nada (1), Un poco (2), Mucho (3). La puntuación total se obtiene sumando las puntuaciones obtenidas en los 18 items. Los items se refieren a consecuencias físicas (p.ej., «Me asusto cuando mi corazón late rápidamente»), cognitivas (p.ej., "Me asusto cuando no puedo concentrarme en los deberes de clase»), y sociales (p.ej., "Me gusta no mostrar mis sentimientos a los demás"). Datos sobre la fiabilidad, validez y estructura factorial de la versión española de la CASI han sido obtenidos por Sandín et al. (en prensa) y Sandín, Chorot, Santed y Valiente (2002).

Cuestionario de Ansiedad Estado/Rasgo para Niños STAIC [State-Trait Anxiety Inventory for Children] (STAIC, Spielberger, 1970). Se aplicó la versión española adaptada por TEA (Spielberger, 1988). Utilizamos la Parte 2, la cual evalúa específicamente el rasgo de ansiedad (p.ej., «Me cuesta enfrentarme a mis problemas", "Me preocupan las cosas del colegio»). Dicha parte consta de 20 items que son contestados por los participantes indicando cómo se sienten habitualmente, según una escala de tres niveles: "Casi nunca» (1), «A veces» (2) y "A menudo" (3).

Cuestionario PANAS (Positive and Negative Affect Schedule) para Niños y Adolescentes, PANASN (Sandín, 1997). Es una forma elaborada por Sandín (1997) para niños y adolescentes a partir de la forma adulta del PANAS de Watson, Clark y Tellegen (1988). El PANASN, al igual que el PANAS, es un cuestionario de autoinforme de 20 elementos. Diez items evalúan el afecto positivo (p.ej., "Soy una persona animada, suelo emocionarme») y otros diez el afecto negativo (p.ej., "Me siento nervioso»). El cuestionario es cumplimentado por el niño/adolescente teniendo en cuenta la manera en que éste se siente y/o comporta habitualmente, siguiendo una escala de tres alternativas de respuesta, descritas éstas como "Nunca» (1), "A veces» (2), y «Muchas veces» (3). Datos sobre la estructura factorial, la validez y la fiabilidad del PANASN han sido presentados por los autores (Romero, Sandín y Chorot, 1997).

Cuestionario de Depresión para Niños, $C D N$ (Sandín y Valiente, 1996). El CDN es 
una prueba de autoinforme elaborada por Sandín y Valiente (1996) con objeto de obtener una evaluación fácil y breve sobre la depresión que experimentan los niños y adolescentes. Más que dirigido a medir el estado de ánimo depresivo, el CDN fue diseñado para evaluar el nivel de depresión clínica. Consta de 16 items, los cuales se refieren a las principales facetas de los síntomas depresivos (anhedonia, ánimo depresivo, desesperanza, inhibición cognitiva, y manifestaciones físicas). La cumplimentación del cuestionario se lleva a cabo teniendo en cuenta la frecuencia de sentimientos, pensamientos o conductas que le hayan ocurrido al sujeto durante las últimas semanas, debiendo señalar, para cada elemento, una entre las tres alternativas de respuesta siguientes: "Nunca» (1), "A veces» (2), o «Muchas veces» (3). Se obtiene una puntuación global en depresión sumando la puntuación obtenida en cada ítem.

\section{RESULTADOS}

Medias, desviaciones típicas

y coeficientes alfa

Antes de exponer los resultados centrales del presente estudio, indicamos en la Tabla 1 algunos resultados generales, esto es, las medias, desviaciones típicas y coe- ficientes alfa de Cronbach correspondientes a los cinco cuestionarios indicados (FSSC-R, CASI, STAIC, PANASN-N, y $\mathrm{CDN}$ ). En términos de la consistencia interna, podríamos decir que todas las pruebas han sido fiables, si bien, como cabría esperar, las pruebas con mayor número de items presentan coeficientes superiores a las pruebas (o subescalas) que poseen menor número de items. Los valores medios sobre intensidad y prevalencia de los miedos están en la línea de los datos preliminares informados previamente por nosotros (Sandín et al., 1998), si bien son ligeramente inferiores a éstos; lo cual podría explicarse porque en nuestro anterior estudio nos basamos en una muestra de menor edad (entre 9 y 11 años). Los resultados, por otra parte, son consistentes con los indicados por otros autores para muestras de niños y adolescentes (p.ej., Ollendick et al., 1991; King et al., 1992; Dong et al., 1994). Otros datos relativos a la intensidad y prevalencia de los miedos comunes en los niños y los adolescentes en función del sexo y la edad puede encontrarlos el lector en Valiente (2001).

Correlaciones entre el nivel de miedos y las variables de predisposición

Con el fin de estudiar la relación entre los miedos (intensidad y prevalencia) y las variables de predisposición calcula-

Tabla 1. Medias, desviaciones típicas y coeficientes alfa (Cronbach) para la muestra total

\begin{tabular}{lccc}
\hline Variable & Media & $D T$ & Alfa \\
\hline FSSC-R (80): & & & \\
$\quad$ Intensidad & 133,5 & 20,5 & 0,94 \\
$\quad$ Prevalencia & 14,7 & 9,5 & \\
CASI (18) & 30,0 & 5,5 & 0,80 \\
STAIC (20) & 37,3 & 7,4 & 0,87 \\
PANASN (20): & & & \\
$\quad$ Afecto positivo (10) & 24,2 & 3,0 & 0,72 \\
Afecto negativo (10) & 20,4 & 3,4 & 0,78 \\
CDN (16) & 26,1 & 4,4 & 0,78 \\
\hline
\end{tabular}

Nota: Entre paréntesis se indica el número de items de cada cuestionario o subescala. 
Tabla 2. Correlaciones de Pearson entre las variables de prevalencia e intensidad de los miedos (FSSC-R) y las variables emocionales de predisposición (muestra total)

\begin{tabular}{|c|c|c|c|c|c|}
\hline FSSC-R & CASI & STAIC & CDN & PANASN-P & PANASN-N \\
\hline \multicolumn{6}{|l|}{ Prevalencia: } \\
\hline Miedos a los animales & 0,34 & 0,33 & $0,22^{*}$ & $-0,11$ & $0,28^{*}$ \\
\hline Miedos al fracaso y a la crítica & 0,38 & 0,38 & $0,31^{\star}$ & $-0,16$ & $0,35^{\star}$ \\
\hline Miedos al peligro y a la muerte & 0,44 & $0,32^{*}$ & $0,21^{*}$ & $-0,03$ & $0,36^{\star}$ \\
\hline Miedos a lo desconocido & 0,40 & $0,36^{*}$ & $0,26^{*}$ & $-0,06$ & $0,32^{\star}$ \\
\hline Miedos médicos & 0,27 & $0,24^{\star}$ & $0,23^{\star}$ & $-0,12$ & $0,22^{\star}$ \\
\hline FSSC-R total & 0,53 & $0,50^{*}$ & $0,38^{\star}$ & $-0,15$ & $0,46^{\star}$ \\
\hline \multicolumn{6}{|l|}{ Intensidad: } \\
\hline Miedos a los animales & 0,41 & $0,38^{*}$ & $0,28^{\star}$ & $-0,13$ & $0,36^{*}$ \\
\hline Miedos al fracaso y a la crítica & 0,49 & $0,43^{*}$ & $0,35^{\star}$ & $-0,17$ & $0,42^{*}$ \\
\hline Miedos al peligro y a la muerte & 0,47 & $0,34^{*}$ & $0,25^{\star}$ & $-0,06$ & $0,39^{*}$ \\
\hline Miedos a lo desconocido & 0,50 & 0,41 * & $0,33^{*}$ & $-0,11$ & $0,45^{*}$ \\
\hline Miedos médicos & 0,36 & $0,30^{*}$ & $0,28^{\star}$ & $-0,13$ & 0,31 * \\
\hline FSSC-R total & 0,61 & 0,54 * & $0,42^{\star}$ & $-0,19$ & $0,53^{*}$ \\
\hline
\end{tabular}

Nota: Para las correlaciones $\geq 0,11, p<0,01$. CASI $=$ Childhood Anxiety Sensitivity Index, STAIC $=$ State-Trait Anxiety Inventory for Children (versión rasgo), $\mathrm{CDN}=$ Cuestionario de Depresión para Niños, PANASN = Positive and Negative Affect Schedule para Niños y Adolescentes ( $\mathrm{P}=$ afecto positivo, $\mathrm{N}=$ afecto negativo). El asterisco $\left.{ }^{\star}\right)$ indica que la correlación es significativamente diferente de la correlación entre la CASI y la misma variable del FSSC-R (transformación Z de Fisher, $p<0,05$ ).

mos los coeficientes de correlación de Pearson entre la prevalencia e intensidad del miedo (prevalencia e intensidad totales y en las diferentes subescalas) y las variables de sensibilidad a la ansiedad (CASI), rasgo de ansiedad (STAIC), afectividad positiva y negativa (PANASN), y depresión (CDN) (véase la Tabla 2) (lógicamente, la afectividad positiva no puede ser considerada como una variable de predisposición a los miedos; su inclusión se efectuó únicamente por razones de control). Las puntuaciones en las cinco dimensiones del FSSC-R se calcularon partiendo de los resultados sobre análisis factoriales exploratorios y confirmatorios establecidos previamente por nosotros (véase Valiente, 2001).

Las correlaciones entre las variables del FSSC-R y las variables de predisposición (Tabla 2) tienden en general a ser más elevadas para la intensidad (rango $=0,25-0,61)$ que para la prevalencia (rango $=0,21-0,53$ ) de los miedos, y superiores para la variable CASI (rango en variables de prevalencia $=0,26-0,53$; rango en variables de intensidad $=0,36$ 0,61 ) que para el resto de variables de predisposición (rango en variables de prevalencia $=0,21-0,50$; rango en variables de intensidad $=0,25-0,54$ ). El rasgo de ansiedad (STAIC) y el afecto negativo (PANASN-N) correlacionan con las variables de miedo de forma muy similar (las correlaciones son casi idénticas), y de forma más elevada que la depresión. Las correlaciones entre el afecto positivo (PANASN-P) y las variables de miedo son prácticamente irrelevantes, y de signo negativo; resultado que cabría esperarse dada la naturaleza de esta variable.

Para contrastar la posible existencia de diferencias estadísticamente significativas entre los coeficientes de correlación de la sensibilidad a la ansiedad y los miedos y los coeficientes de las restantes variables de predisposición, calculamos la $Z$ transformada de Fisher. Mediante este estadístico, comparamos dos a dos, y para cada variable de miedo, la correlación de la CASI y la correlación de cada 
una de las restantes variables de predisposición. Como indicamos en la Tabla 2; en todas las comparaciones resultaron significativas las diferencias excepto en dos [correlaciones del rasgo de ansiedad (STAIC) con la prevalencia de miedos a los animales y miedos al fracaso y a la crítica].

\section{DISCUSIÓN}

En este estudio, basándonos en una muestra amplia de niños y adolescentes, hemos examinado la relación entre los miedos autoinformados (intensidad y prevalencia) y un conjunto de variables de diferencias individuales, potencialmente de predisposición hacia los miedos (i.e., sensibilidad a la ansiedad, rasgo de ansiedad, afectividad negativa y depresión). Basándonos en la evidencia de la literatura científica asumíamos la existencia de: (1) una relación positiva entre la intensidad y/o prevalencia de los miedos y las variables de predisposición general, i.e., el rasgo de ansiedad, la depresión, y el afecto negativo; y (2) una relación más específica entre los miedos y la sensibilidad a la ansiedad que entre éstos y las variables de predisposición general.

Los análisis correlacionales que hemos presentado en la sección de resultados aportan información consistente con nuestra hipótesis, tanto para las medidas de prevalencia como de intensidad de los miedos (si bien, las correlaciones, como cabría esperarse dada la naturaleza de las variables, tienden a ser siempre superiores para la intensidad que para la prevalencia). Los resultados indican que las correlaciones entre el miedo y las variables de predisposición general son todas relevantes y estadísticamente significativas, siendo más modestas con la depresión que con las otras dos variables de predisposición general (rasgo de ansie- dad y afecto negativo). Estos resultados apoyan la evidencia presentada por algunos autores que han aportado correlaciones moderadas y positivas entre la ansiedad y el nivel de miedo en poblaciones de niños y adolescentes (King et al., 1992; Ollendick, 1983; Scherer y Nakamura, 1968), así como también la existencia de correlaciones más modestas entre el miedo y la depresión que entre el miedo y la ansiedad (Dong et al., 1994; Ollendick et al., 1991; Muris et al., 2001).

Estos autores han sugerido que las correlaciones positivas que exhiben la ansiedad y la depresión con los niveles de miedo podrían justificar la existencia del constructo de afectividad negativa (Dong et al., 1994; Ollendick et al., 1991), constructo de distress invocado con frecuencia para explicar el factor general de los miedos. Nuestros datos, a este respecto, indican que las correlaciones entre el afecto negativo y los miedos son muy similares a las correlaciones entre el rasgo de ansiedad y los miedos. Por tanto, el factor general de distress que subyace a todos los miedos (¿afecto negativo?) parece ser más similar al rasgo de ansiedad que a la depresión. Es probable que el afecto negativo sea más un factor común a los miedos, los trastornos de ansiedad y la depresión que un factor general específico de los miedos (Zinbarg y Barlow, 1996). Por otra parte, las correlaciones nulas o bajas (y negativas) entre el miedo y el afecto positivo proporcionan apoyo empírico a la validez divergente de este constructo y a su diferenciación del afecto negativo; así mismo, son consistentes con la evidencia de la literatura sobre la naturaleza de los constructos de afecto positivo y negativo (Watson et al., 1988; Sandín 1997).

Aunque se ha discutido bastante sobre la identidad del factor general común a todos los miedos, algunos autores han sugerido expresamente que éste podría ser el rasgo de ansiedad 
(Taylor, 1995) o bien la sensibilidad a la ansiedad (Sandín, 1997, 1999b). Los datos del presente estudio apoyan más bien la hipótesis sugerida por este último autor, ya que las correlaciones entre la sensibilidad a la ansiedad y el miedo son siempre superiores que las correlaciones que exhibe éste con el rasgo de ansiedad (o con cualquier otra variable de predisposición general). Como hemos documentado en la Tabla 2, tales correlaciones son significativamente diferentes entre sí, y afectan tanto a las puntuaciones en la escala total como a las diferentes dimensiones de los miedos, y tanto a las puntuaciones de intensidad como a las de prevalencia. Por tanto, basándonos en estos resultados, podríamos concluir diciendo que, mientras el rasgo de ansiedad y/o el afecto negativo constituyen factores generales de predisposición a los problemas de ansiedad (incluidos los miedos) y a la depresión, la sensibilidad a la ansiedad es un factor general de predisposición específica para los miedos. Los resultados, por otra parte, apoyan la hipótesis sugerida en la literatura reciente sobre el valor de la sensibilidad a la ansiedad como factor esencial de vulnerabilidad hacia los miedos y otros problemas de ansiedad, tanto en población adulta (McNally, 1994; Sandín et al., 1996; Taylor, 1995) como en población infantojuvenil (Silverman y Weems, 1999; Sandín et al., en prensa).

\section{REFERENCIAS BIBLIOGRÁFICAS}

Boyd, C.P., y Gullone, E. (1997). An investigation of negative affectivity in Australian adolescents. Journal of Clinical Child Psychology, 26, 190-197.

Chorot, P., Sandín, B., Valiente, R.M., Santed, M.A., y Romero, M. (1997). Actitud hacia la enfermedad, ansiedad y sintomatología somática en pacientes con trastorno de pánico e hipocondría. Revista de Psicopatología y Psicología Clínica, 2, 123-136.

Chorpita, B.F., y Daleiden, E.L. (2000). Properties of the Childhood Anxiety Sensitivity Index in children with anxiety disorders: Autonomic and nonautonomic factors. Behavior Therapy, 31, 327-349.

Chorpita, B.F., Albano, A.M., y Barlow, D.H. (1996). Child Anxiety Sensitivity Index: Considerations for children with anxiety disorders. Journal of Clinical Child Psychology, 25, 77-82.

Chorpita, B.F., Albano, A.M., y Barlow, D.H. (1998). The structure of negative emotions in a clinical sample of children and adolescents. Journal of Abnormal Psychology, 107, 74-85.

Dong, Q., Yang, B., y Ollendick, T.H. (1994). Fears in Chinese children and adolescents and their relations to anxiety and depression. Journal of Child Psychology and Psychiatry, 35, 351-363.

Gullone, E., King, N.J., y Ollendick, T.H. (2001). Self-reported anxiety in children and adolescents: A three-year follow-up study. Journal of Genetic Psychology, 162, 5-19.

Joiner, T.E., Catanzaro, S.J., y Laurent, J. (1996b). Tripartite structure of positive and negative affect, depresión, and anxiety in child and adolescent psychiatric inpatients. Journal of Abnormal Psychology, 105, 401-409.

Joiner, T.E.,Jr., Catanzaro, S.J., Laurent, J., Sandín, B., y Blalock, J.A. (1996a). Modelo tripartito sobre el afecto positivo y negativo, la depresión y la ansiedad: Evidencia basada en la estructura de los síntomas y en diferencias sexuales. Revista de Psicopatología y Psicología Clínica, 1, 27-34.

King, N.J., Gullone, E., y Ollendick, T.H. (1992). Manifest anxiety and fearfulness in children and adolescents. The Journal of Genetic Psychology, 153, 63-73.

King, N.J., Ollendick, T.H., y Gullone, E. (1991). Negative affectivity in children and adolescents: Relations between anxiety and depression. Clinical Psychology Review, 11, 441-459.

Lau, J.L., Calamari, J.E., y Waraczynski, M. (1996). Panic attack symptomatology and anxiety sensitivity in adolescents. Journal of Anxiety Disorders, 10, 355-364. 
McNally, R.J. (1994). Panic Disorder: A critical analysis. New York: Guilford Press.

Muris, P., Schmidt, H., Merckelbach, H., y Schouten, E. (2001). The structure of negative emotions in adolescents. Journal of Abnormal Child Psychology, 29, 331-337.

Ollendick, T.H. (1983). Reliability and validity of the Revised Fear Survey Schedule for Children (FSSC-R). Behaviour Research and Therapy, 21, 685-692.

Ollendick, T.H., Yule, W., y Ollier, K. (1991). Fears in British Children and their relationship to Manifest Anxiety and Depression. Journal of Child Psychology and Psychiatry, 32, 321-331.

Romero, M., Sandín, B., y Chorot, P. (1997. abril). Datos preliminares con el cuestionario de afecto positivo y negativo para niños y adolescentes (PANASN). Póster presentado al I Congreso de la Asociación Española de Psicología Clínica y Psicopatología, Madrid.

Sandín, B. (1997). Ansiedad, miedos y fobias en niños y adolescentes. Madrid: Dykinson.

Sandín, B. (1999a). Delimitaciones conceptuales. En B. Sandín (Ed.), Las fobias específicas (pp. 13-24). Madrid: UNED-FUE.

Sandín, B. (1999b). Diagnóstico y fenomenología. En B. Sandín (Ed.), Las fobias específicas (pp. 48-53). Madrid: Klinik.

Sandín, B., y Chorot, P. (1998). Dimensiones de los miedos en niños: Estructura factorial del FSSC-R. Psiquis, 19, 23-32.

Sandín, B., Chorot, P., y McNally, R.J. (1996). Validation of the Spanish version of the Anxiety Sensitivity Index in a clinical sample. Behaviour Research and Therapy, 34, 283-209.

Sandín, B., Chorot, P., y McNally, R.J. (2001). Anxiety sensitivity index: Normative data and its differentiation from trait anxiety. Behaviour Research and Therapy, 39, 213219.

Sandín, B., Chorot, P., Santed, M.A., y Valiente, R.M. (2002). Análisis factorial confirmatorio del Índice de Sensibilidad a la
Ansiedad para niños. Psicothema, 14, 333339.

Sandín, B., Chorot, P., Valiente, R.M., y Santed, M.A. (1998). Frecuencia e intensidad de los miedos en los niños: Datos normativos. Revista de Psicopatología y Psicología Clínica, 3, 15-25.

Sandín, B., Chorot, P., Valiente, R.M., y Santed, M.A. (en prensa). Sensibilidad a la ansiedad en niños y predicción del nivel de miedos. Psicología Conductual.

Sandín, B., y Valiente, R.M. (1996). Cuestionario de Depresión en Niños. Madrid: Universidad Nacional de Educación a Distancia (policopiado).

Scherer, M.W., y Nakamura, C.Y. (1968). A fear survey schedule for children. Behaviour Research and Therapy, 6, 172-182.

Silverman, W.K., y Weems, C.F. (1999). Anxiety sensitivity in children. En S. Taylor (Ed.), Anxiety sensitivity: Theory, research, and treatment of the fear of anxiety (239-268). Mahwah, NJ: LEA.

Silverman, W.K., Fleisig, W., Rabian, B., y Peterson, R.A. (1991). Childhood Anxiety Sensitivity Index. Journal of Clinical Child Psychology, 20, 162-168.

Spielberger, C.D. (1988). Cuestionario de Ansiedad Estado/Rasgo en Niños (STAIC). Madrid: TEA (orig. inglés 1970).

Taylor, S. (1995). Anxiety sensitivity: Theoretical perspectives and recent findings. Behaviour Research and Therapy, 33, 243258.

Valiente, R.M. (2001). Estructura y adquisición de los miedos en la infancia y la adolescencia. UNED, Madrid (Tesis Doctoral, no publicada).

Watson, D., Clark, L.A., y Tellegen, A. (1988). Development and validation of brief measures of positive and negative affect: The PANAS scales. Journal of Personality and Social Psychology, 54, 1063-1070.

Zinbarg, R.E., y Barlow, D.H. (1996). Structure of anxiety and the anxiety disorders: A hierarchical model. Journal of Abnormal Psychology, 105, 181-193. 